\title{
GI-MS46-P15 | BIological USER Support AT THE EUROPEAN XFEL
}

Schubert, Robin (European XFEL, Schenefeld, GER); Han, Huijong (European XFEL, Schenefeld, GER); Meza, Domingo (European XFEL, Schenefeld, GER); Round, Ekaterina (EMBL Hamburg Outstation, Hamburg, GER); Schulz, Joachim (European XFEL, Schenefeld, GER); Lorenzen, Kristina (European XFEL, Schenefeld, GER)

The European XFEL Sample Environment and Characterization group provides in-house expertise in sample preparation, delivery and diagnostics methods for the six scientific instruments at European XFEL. The group operates the user sample preparation laboratories and aims to provide excellent support for users coming for beam-time. This includes supporting them with state-of-the-art sample preparation and characterization methods as well as the support and development of novel sample delivery techniques.

The Sample Environment and Characterization group provides large scale instrumentation like X-ray generators, electron microscopes and vacuum test chambers and is involved in the fields of surface science, fluidic systems, cryogenics, magnetism, chemistry, and biology to provide assistance for users preparing their samples and bringing their samples into the beam.

The XBI User Consortium operates sample preparation and characterization facilities for biological samples at the European XFEL. This purpose built laboratory offers a wide range of biochemical and biophysical characterization techniques. The Sample Environment and Characterization and XBI staff can support users in all steps from gene expression through protein purification, biophysical characterization, and crystallization up to sample delivery of both crystalline and non-crystalline samples of a wide range of biological systems at the instruments of the European XFEL.

The poster will present an overview about available equipment in the labs. In addition, possible workflows for sample preparation and characterization are combined and visualized. It serves as a roadmap to optimize samples step by step in order to ensure the best sample quality for experiments at European XFEL. 